\section{A New Bat-HKU2-like Coronavirus in Swine, China, 2017}

\section{Lang Gong, ${ }^{1}$ Jie Li, ${ }^{1}$ Qingfeng Zhou, Zhichao Xu, Li Chen, Yun Zhang, Chunyi Xue, Zhifen Wen, Yongchang Cao}

Author affiliations: State Key Laboratory of Biocontrol, Life Sciences School, Sun Yat-sen University, Guangzhou, China (L. Gong, J. Li, Z. Xu, L. Chen, Y. Zhang, C. Xue, Z. Wen, Y. Cao); Guangdong Wen's Foodstuffs Group Co., Ltd., Yunfu, China

(J. Li, Q. Zhou, L. Chen, Z. Wen)

DOI: https://doi.org/10.3201/eid2309.170915

We identified from suckling piglets with diarrhea in China a new bat-HKU2-like porcine coronavirus (porcine enteric alphacoronavirus). The GDS04 strain of this coronavirus shares high aa identities (>90\%) with the reported batHKU2 strains in Coronaviridae-wide conserved domains, suggesting that the GDS04 strain belongs to the same species as HKU2.

Q everal pathogens are thought to be responsible for $\checkmark$ porcine diarrhea, including porcine epidemic diarrhea virus (PEDV) (1), transmissible gastroenteritis virus (2), porcine deltacoronavirus (3), porcine group A rotavirus (4), and emerging viruses like porcine kobuvirus (5). To add to the list, we have identified from suckling piglets with diarrhea in China a new bat-HKU2-like porcine coronavirus (porcine enteric alphacoronavirus [PEAV]).

Since December 2010, large-scale outbreaks of diarrhea in suckling piglets have been reported across China (1), and vaccination against PEDV has been relatively effective for diarrhea prevention. However, in February 2017, outbreaks of severe diarrhea occurred in swine herds vaccinated against PEDV in Guangdong, China. All ill pigs showed severe watery diarrhea, and their clinical onset occurred a few days later than those infected with PEDV. In initial tests with reverse transcription PCR using specific primers for PEDV, transmissible gastroenteritis virus, porcine group A rotavirus, or porcine deltacoronavirus, none of these viruses could be detected in all clinical samples. Furthermore, the recovered sows showed no seroneutralizing antibodies against PEDV.

To investigate the possible causative pathogen or pathogens causing this recent severe diarrhea in suckling piglets, we obtained excreta from 32 ill newborn piglets from 3 farms. We divided 5-day-old piglets into 4 groups of 5 each (3 groups according to the origin of the excreta, plus

${ }^{1}$ These authors contributed equally to this article.
1 control group). We inoculated each animal with $5 \mathrm{~mL}$ of excreta through the oral route. After 2 days, all inoculated animals exhibited similar clinical symptoms, including diarrhea and dehydration. We randomly selected 2 inoculated pigs in each group and performed necropsies on days 3 and 5 postinoculation, respectively. We filtrated homogenate of small intestine and intestinal contents, using the resultant supernatant for RNA extraction as described previously (2). We extracted total RNA by using a TRIzol reagent (Invitrogen Life Technologies, Grand Island, NY, USA) and eliminated ribosomal RNA with the Ribo-Zero rRNA Removal Kit (Illumina, San Diego, CA, USA) according to the manufacturer's instructions. After reverse transcription PCR with random primers, we performed 150-bp pairedend shotgun metatranscriptome sequencing on the cDNA libraries by using an Illumina HiSeq system.

After assembling and mapping sequencing reads, we obtained a complete genome sequence of the PEAV GDS04 strain, which we then deposited in GenBank (accession no. MF167434). The full length of the PEAV genome is 27,171 nt (excluding the poly-A tail); this size is similar to that of bat-like HKU2 strains of coronavirus (6). The full genome of PEAV GDS04 strain shares high nucleotide identities $(\approx 95 \%)$ with the reported bat-HKU2 strains.

Our phylogenetic analysis based on the whole genome of GDS04 and representatives of 4 established coronavirus genera, including the human and bat-like coronaviruses, demonstrated that GDS04 clusters with bat-like coronaviruses (Figure). According to the phylogenetic tree, the position of GDS04 is between HKU2 and BtRf alphacoronavirus. The HKU2 strain was identified in Hong Kong and Guangdong Province, China, in 2007 (6), and the BtRf alphacoronavirus strain was detected in China in 2012 (http:// www.ncbi.nlm.nih.gov/nuccore/NC_028824.1). Both viruses are bat-associated viruses. However, the 2 strains are distributed in relatively different branches (Figure). Based on a comprehensive comparative analysis of the genomes of various groups of coronaviruses, we classified GDS04 as an alphacoronavirus. Our sequence analysis revealed that GDS04 has $80 \%$ nt and $87 \%$ aa identity with the spike (S) protein of the HKU2 strain. Moreover, the $\mathrm{S}$ protein of the GDS04 genome is 6 bp longer than the S protein in the HKU2 strain, which has the smallest $\mathrm{S}$ protein among all coronaviruses. Nevertheless, the GDS04 strain shares 95.7\% aa identity with HKU2 in nonstructural protein (nsp) 3 (adenosine diphosphate-ribose 1'-phosphatase), 96.4\% in nsp5 (3C-like protease), 94.6\% in nsp12 (RNA-dependent RNA polymerase), $99.8 \%$ in nsp13 (helicase), $99.2 \%$ in nsp14 (3'-to-5' exonuclease), 99.4\% in nsp15 (poly[U]specific endoribonuclease), and $97.6 \%$ in nsp16 (2'-O-ribose methyltransferase). These nonstructural proteins are Coronaviridae-wide conserved domains in replicase polyprotein pplab. A threshold of $>90 \%$ in aa sequence identity 


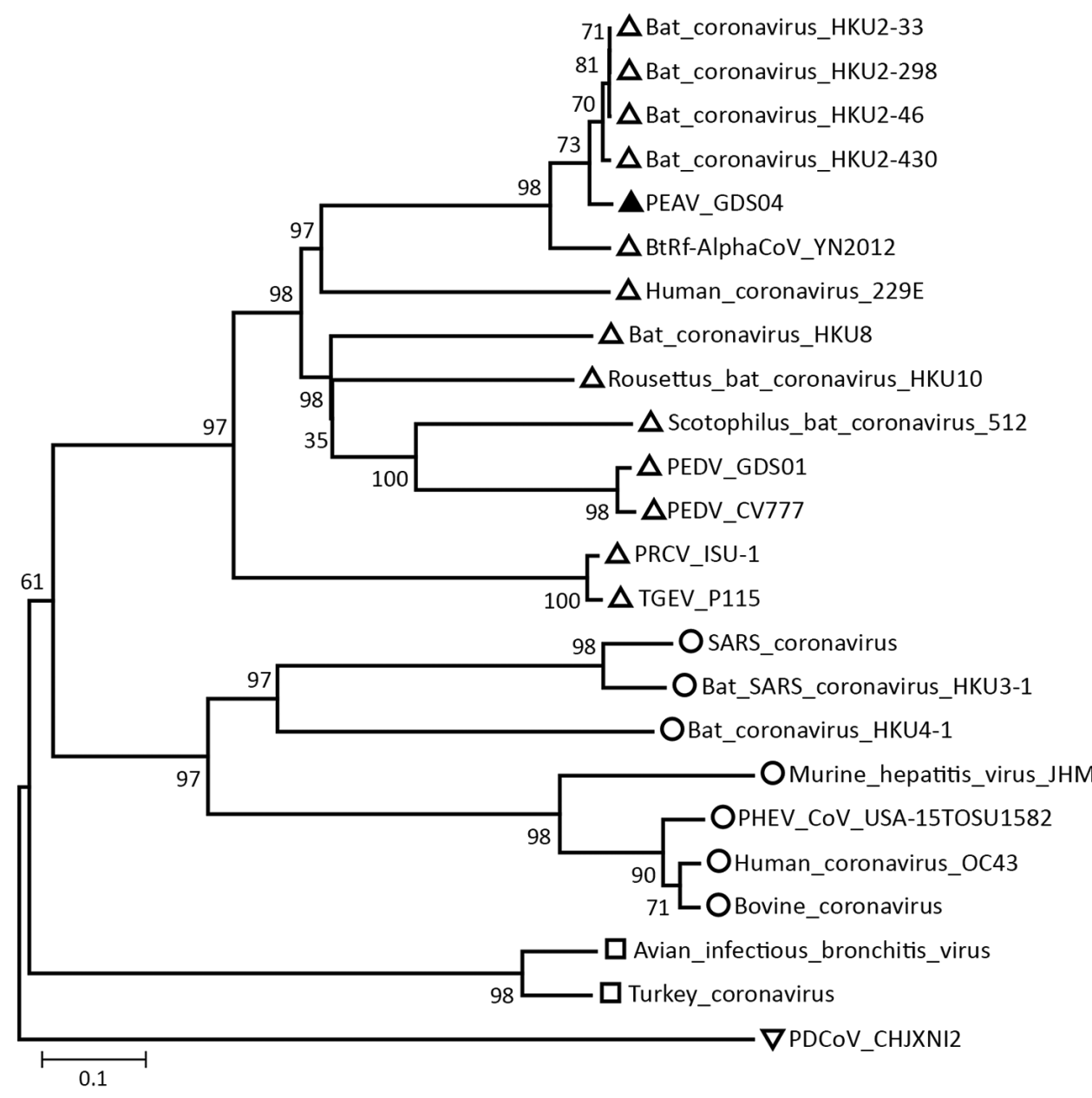

Figure. Phylogenetic tree based on the whole-genome sequences of PEAV, bat CoVs, and other representative CoVs, China, 2017. Analyses were conducted by using MEGA software version 6.0 (http://www.megasoftware. net) with the neighborjoining algorithm. Bootstrap values were calculated with 1,000 replicates. The number on each branch indicates bootstrap values. Solid triangle indicates the GDS04 strain, open triangles alphacoronaviruses, circles betacoronaviruses, squares gammacoronavirus, inverted triangle deltacoronavirus. Scale bar indicates nucleotide substitutions per site. CoV, coronavirus; PDCoV, porcine deltacoronavirus; PEAV, porcine enteric alphacoronavirus; PEDV, porcine epidemic diarrhea virus; PHEV, porcine hemagglutinating encephalomyelitis virus; PRCV, porcine respiratory coronavirus; SARS, severe acute respiratory syndrome; TGEV, transmissible gastroenteritis virus. suggests that 2 viruses are of the same species; our findings suggest that the GDS04 strain belongs to the same species as HKU2.

Furthermore, we designed specific primers of the $n$ gene for the detection of the GDS04 strain. By using reverse-transcription PCR with these primers, we found that 97 out of 308 clinical intestinal or fecal samples were positive for GDS04. We collected all clinical samples from 25 farms in Guangdong Province during February-April 2017 and used 20 samples collected from healthy vaccinated piglets as negative controls.

In summary, we report preliminary data on our detection of a new coronavirus-like virus, PEAV. PEAV is thought to be responsible for the most recent diarrhea endemic in pig herds in southern China. Virus isolation and serologic testing are under way. The outbreak of the newly discovered virus arose among swine with severe diarrhea in swine breeding farms in southern China, suggesting the regional outbreaks of diarrhea could contribute to the emergence of new pandemic viruses. Extensive surveillance for GDS04 PEAV is required to define its epidemiology and evolution.
This work was supported by the National Key Research and Development Program (grant no. 2016YFD0500101).

The animal study was supervised by the Institutional Animal Care and Use Committee of the Sun Yat-sen University (approval no. IACUC DD-17-0403) and used in accordance with regulation and guidelines of this committee.

Mr. Gong is a PhD student in the life sciences school at Sun Yat-sen University. His primary research interests include the molecular epidemiology of novel coronaviruses and influenza vaccine. Dr. Li is a postdoctoral fellow in the life sciences school at Sun Yat-sen University. His research focuses on bioinformatics, genomics and molecular epidemiology of coronaviruses.

\section{References}

1. Sun RQ, Cai RJ, Chen YQ, Liang PS, Chen DK, Song CX. Outbreak of porcine epidemic diarrhea in suckling piglets, China. Emerg Infect Dis. 2012;18:161-3. http://dx.doi.org/10.3201/ eid1801.111259

2. Pensaert MB, de Bouck P. A new coronavirus-like particle associated with diarrhea in swine. Arch Virol. 1978;58:243-7. http://dx.doi.org/10.1007/BF01317606 
3. Dong N, Fang L, Zeng S, Sun Q, Chen H, Xiao S. Porcine deltacoronavirus in mainland China. Emerg Infect Dis. 2015;21:2254-5. http://dx.doi.org/10.3201/eid2112.150283

4. Theuns S, Vyt P, Desmarets LM, Roukaerts ID, Heylen E, Zeller M, et al. Presence and characterization of pig group A and C rotaviruses in feces of Belgian diarrheic suckling piglets. Virus Res. 2016;213:172-83. http://dx.doi.org/10.1016/j.virusres.2015.12.004

5. Reuter G, Boros A, Pankovics P. Kobuviruses - a comprehensive review. Rev Med Virol. 2011;21:32-41. http://dx.doi.org/10.1002/ rmv. 677

6. Lau SK, Woo PC, Li KS, Huang Y, Wang M, Lam CS, et al. Complete genome sequence of bat coronavirus HKU2 from Chinese horseshoe bats revealed a much smaller spike gene with a different evolutionary lineage from the rest of the genome. Virology. 2007;367:428-39. http://dx.doi.org/10.1016/ j.virol.2007.06.009

Address for correspondence: Zhifen Wen, Guangdong Wen's Group Academy, Guangdong Wen's Foodstuffs Group Co., Ltd., Yunfu 527400, China; email: wenzhifen@163.com; Yongchang Cao, Life Sciences School, Sun Yat-sen University, Higher Education Mega Center, Guangzhou 510006, China; email: caoych@mail.sysu.edu.cn

\section{Antimicrobial Drug-Resistant Shiga Toxin-Producing Escherichia coli Infections, Michigan, USA}

\author{
Sanjana Mukherjee, Rebekah E. Mosci, \\ Chase M. Anderson, Brian A. Snyder, \\ James Collins, James T. Rudrik, \\ Shannon D. Manning
}

Author affiliations: Michigan State University, East Lansing, Michigan, USA (S. Mukherjee, R.E. Mosci, C.M. Anderson,

B.A. Snyder, S.D. Manning); Michigan Department of Health and Human Services, Lansing (J. Collins, J.T. Rudrik)

DOI: https://doi.org/10.3201/eid2309.170523

High frequencies of antimicrobial drug resistance were observed in $\mathrm{O} 157$ and non-O157 Shiga toxin-producing E. coli strains recovered from patients in Michigan during 2010-2014. Resistance was more common in non-O157 strains and independently associated with hospitalization, indicating that resistance could contribute to more severe disease outcomes.

Shiga toxin-producing Escherichia coli (STEC) contributes to 265,000 cases of foodborne illness annually in the United States (1). Most infections are caused by O157 strains; however, non-O157 STEC infections have increased (2). Antimicrobial drug resistance among STEC has been reported (3-5) but is probably underestimated. Given the importance of resistance in E. coli pathotypes, we sought to determine the prevalence of resistant STEC infections and assess the effects of resistance on disease.

We obtained 358 STEC isolates from the Michigan Department of Health and Human Services (MDHHS) Reference Laboratory (Lansing, MI, USA), collected during 2010-2014. Of these, 14 were outbreak associated. We examined 1 strain per outbreak using protocols approved by Michigan State University (MSU; Lansing, MI, USA; IRB \#10-736SM) and MDHHS (842-PHALAB). Overall, 31 (8.8\%) strains (23 non-O157, 8 O157) were resistant to antimicrobial drugs (Table). Resistance to ampicillin (7.4\%) was most common, followed by trimethoprim/sulfamethoxazole (SXT) $(4.0 \%)$ and ciprofloxacin $(0.3 \%)$. Compared with national rates, resistance to ampicillin and SXT was higher, but not significantly different, for O157 isolates from Michigan (online Technical Appendix Figure 1, https://wwwnc. cdc.gov/EID/article/23/9/17-0523-Techapp1.pdf) (6). One strain was resistant to all drugs, and all resistant strains had high MICs (ampicillin, $>64 \mu \mathrm{g} / \mathrm{mL}$; ciprofloxacin, $>32 \mu \mathrm{g} /$ $\mathrm{mL}$; SXT, in 1:19 ratio, $>32 / 608 \mu \mathrm{g} / \mathrm{mL}$ ). Notably, resistance was twice as common for non-O157 (11.1\%) than for O157 $(5.5 \%)$ strains. O111 strains $(\mathrm{n}=7)$ had significantly higher resistance frequencies $(24.1 \%)$ than other non-O157 serogroups $(\mathrm{p}=0.03)$. We found variation by year and season; resistance frequencies were highest in 2012 (online Technical Appendix, Figure 2) and during winter/spring (online Technical Appendix Table 1), but neither trend was significant. We also observed a strong but nonsignificant association between resistance and hospitalization but no association for urban versus rural residence (7) or county after stratifying by prescription rates $(8)$ in the univariate analyses.

We conducted a multivariate analysis using logistic regression, with hospitalization as the dependent variable; we included variables with significant $(\mathrm{p} \leq 0.05)$ and strong $(p \leq 0.20)$ associations from the univariate analysis as independent variables. Forward selection indicated that hospitalized patients were more likely to have resistant infections (odds ratio [OR] 2.4, 95\% CI 1.00-5.82) and less likely to have non-O157 infections (OR $0.4,95 \%$ CI 0.21-0.61) (online Technical Appendix Table 2), suggesting that resistant infections or O157 infections may cause more severe clinical outcomes. Patients $\geq 18$ years of age, women, and patients with bloody diarrhea were also more likely to be hospitalized.

Although we found no significant difference by stx profile, strains possessing stx 1 only were more commonly resistant than strains with stx2 alone $(\mathrm{p}=0.27$ by Fisher exact test). All 23 (100\%) resistant non-O157 STEC and 1 